1. MBBS, FCPS

PAC Hospital, Karma

2. MBBS

PAC Hospital, Karma

3. MBBS, FCPS

PAC Hospital, Karma

Correspondence Address:

Dr. Muhammad Tahir

MBBS, FCPS

PAC HOSPITAL KARMA

dr_tahir777@yahoo.com

Article received on:

18/02/2016

Accepted for publication:

21/06/2016

Received after proof reading: 08/08/2016

\title{
NONULCER DYSPEPSIA; \\ FREQUENCY IN YOUNG PATIENTS AND ITS RESPONSE TO HELICOBACTER PYLORI ERADICATION AND TRIAL OF PROTON PUMP INHIBITORS \\ dr_tahir777@yahoo.com \\ Dr. Farrukh Zahra', Dr Muhammad Younis², Dr. Uzma Nisar ${ }^{3}$
}

ABSTRACT... Objectives: To determine the frequency of nonulcer dyspepsia in patients presenting with the peptic ulcer like symptoms and to determine the effect of $\mathrm{H}$. pylori eradication on dyspeptic symptoms and the effect of six weeks trial of proton pump inhibitor as treatment measure. Study design: Cross-sectional study. Setting: Combined Military hospital Quetta, Department of medicine. Period: Six months from Oct, till March 2009. Subjects and Methods: Patients coming to medicine department with complaints of dyspepsia. Results: Total no of patients were 145 out of which male were 90 while 55 were females. The mean age was 29.68 \pm 4.53 . These subjects were given $\mathrm{H}$ pylori eradication therapy and response was graded as patients responded or no response. 89 subjects $(61.4 \%)$ responded to $\mathrm{H}$ pylori eradication treatment while 56 subjects $(38.6 \%)$ showed no symptomatic benefit. Non responder to eradication i.e., 37 males and 36 females were given further six week treatment with omeprazole, and subjective response to treatment was graded as complete resolution found in 31 patients ( $21.4 \%$ ), while partial resolution was present in 15 patients (10.3\%). 07 patients $(4.8 \%)$ showed no improvement while 03 patients $(2.1 \%)$ showed worsening of symptoms. Conclusions: The prevalence of non-ulcer dyspepsia is high among symptomatic individuals. The response to $\mathrm{H}$ pylori eradication was significant. Non responders also showed considerable symptomatic benefit with PPI. Therefore, individuals with recurrent dyspeptic symptoms should be given trial of $\mathrm{H}$. pylori eradication.

Key words: Non ulcer dyspepsia, H. pylori, proton pump inhibitor, effect, functional dyspepsia, treatment

Article Citation: Zahra F, Younis M, Nisar U. Nonulcer dyspepsia; frequency in young patients and its response to helicobacter pylori eradication and trial of proton pump inhibitors. Professional Med J 2016;23(8):907-911. DOI: 10.17957/ TPMJ/16.2941

\section{INTRODUCTION}

Non ulcer Dyspepsia means chronic persistent or recurrent pain or discomfort in the central upper abdomen which originates in gastrointestinal tract with no identifiable cause in routine clinical and diagnostic workup. ${ }^{1}$ The main type of dyspepsia seen in primary care and gastroenterology clinics is NUD also called as functional dyspepsia, that is no known etiology can be found for the symptoms and causes for dyspepsia such as duodenal ulcer, gastric ulcer, esophagitis (inflamed esophagus), gastritis (inflamed stomach), etc., are not the cause. ${ }^{2}$ So functional dyspepsia is one of the most frequent reason for consultation in clinical practice. NUD is a diagnosis of exclusion and up to $60 \%$ of all investigated patients will have NUD. ${ }^{3}$ The cause of non-ulcer dyspepsia is not clear but is thought to be heterogeneous. The underlying pathophysiology of NUD is associated with motility and non-motility disorders $(\mathrm{H}$ pylori infection) and psychosocial factors. The patients mostly present with nonspecific symptoms i.e. bloating, belching, flatulence, excessive fullness after eating and nausea. Symptoms are mostly intermittent. Psychological conditions such as anxiety; stress and depression plays an important role in the recurrence of symptoms. ${ }^{4}$

Upper Gl endoscopy is the main diagnostic intervention to determine the etiology of dyspepsia. This is particularly important for patients who presents with alarm symptoms and signs i.e. weight loss, anemia, upper Gl bleed, positive fecal occult blood suggestive of underlying organic condition before one makes the diagnosis of NUD. ${ }^{5}$ The management of the 
disorder is unsatisfactory, and there are few studies showing that active treatment is better than a placebo.

Studies have been done to show the possible association of $\mathrm{H}$. Pylori infection causing NUD (as in ulcer disease). ${ }^{6}$ However there is controversy regarding role of $\mathrm{H}$ pylori in NUD, study and treatment result have also been found inconsistent..$^{7-9}$ Although meta-analysis of 10 studies did not demonstrate any improvement in symptoms with eradication therapy but an updated review of 17 trials confirmed small but statistically significant benefit. ${ }^{10}$

NUD is responsible for significant burden on health care system. It has financial impact in terms of money spent on investigations and medication. It also causes reduced productivity at work place and thus has psychological impact as well. A better understanding of this disease and its treatment can improve patient care and result in reduction of unnecessary medical costs. Empirical helicobacter pylori eradication therapy is not only economical but quite safe as well. However, attention must be paid to rule out any organic cause.

As there is a limited data regarding response to $\mathrm{H}$ pylori eradication therapy in patients with NUD and symptomatic relief with PPI in our population, depending upon the results of our study, we will be able to recommend / refute $\mathrm{H}$. pylori eradication as treatment measure in patients with NUD. Also based on the study results in non-responders, we will be able to support / refute the use of PPI for symptomatic benefit in patients with NUD.

\section{Objectives}

1. To determine the frequency of non-ulcer dyspepsia in patients presenting with the peptic ulcer like symptoms.

2. To determine the effect of $\mathrm{H}$. pylori eradication on dyspeptic symptoms in the patients with NUD.

3. To determine the effect of six weeks trial of proton pump inhibitor (RPI) as treatment measure for NUD.
Study design

Cross-sectional study.

\section{Setting}

Combined Military hospital Quetta, Department of medicine.

\section{Duration of study}

Six months from Oct, till March 2009.

\section{Subjects and Methods}

Using non probability consecutive sampling, 145 subjects including both genders less than 55 years with 12 weeks history of dyspepsia were included in study. Person with alarm symptoms and signs, smokers and those using NSAIDS were excluded to reduce bias in study results. Symptomatic individuals visiting medical OPD, CMH Quetta was included in the study after informed consent. Data on clinical assessment was recorded on pre-designed assessment preformed as per annex $\mathrm{A}$. Response to $\mathrm{H}$ pylori eradication was assessed. Those who did not respond were given six week treatment with omeprazole and response was graded as complete, partial, no improvement or Worsening of symptom. Mean and SD was calculated for age and hours off work. Frequencies and percentages were presented for individual dyspeptic symptoms including daily symptom, severity of symptom, response to $\mathrm{H}$. pylori eradication and treatment with proton pump inhibitor.

\section{RESULTS}

Total no of patients was 145 out of which male patients were 90 while 55 female were also included. The mean age was $29.68 \pm 4.53$. These subjects were given $\mathrm{H}$ pylori eradication and response to eradication therapy was graded as patients responded or no response. We found that out of 145,89 subjects $(61.4 \%)$ responded to $\mathrm{H}$ pylori eradication treatment while 56 subjects (38.6\%) showed no symptomatic benefit. So total 53 males and 36 females responded to eradication. Non responder to eradication i.e., 37 males and 36 females were given further six week treatment with omeprazole, and subjective response to treatment was graded as complete resolution found in 31 patients $(21.4 \%)$, while partial 
resolution was present in 15 patients (10.3\%). 07 patients $(4.8 \%)$ showed no improvement while 03 patients $(2.1 \%)$ showed worsening of symptoms. Frequency of daily symptoms was seen in 56 patients (38.6\%). While 42 patients (28.96\%) had occasional symptoms. Mild symptoms were present in 73 patients $(50.34 \%)$, moderate in 47 patients $(32.4 \%)$ and severe symptoms in 25 cases (17.2\%). Regarding hours off work, mean was calculated as $1.45 \pm 1.2$

\section{DISCUSSION}

Dyspepsia is relatively common disorder, with a prevalence of between $30 \%$ and $40 \%$ in much population survey. There is no data available that shows precise prevalence of NUD in Pakistan however, Studies that were conducted in different centers on dyspeptic patient revealed that the incidence of NUD ranges from $28 \%$ to $59 \%$ in both genders. ${ }^{4-5}$

Our study results are in accordance with those of an earlier randomized control trial showing symptomatic improvement from eradicating $\mathrm{H}$. pylori in NUD. ${ }^{3-11}$

In our study, non-responder to eradication therapy was found to be $38.4 \%$ and these patients after six weeks treatment with PPI resulted in "partial resolution" in $10.3 \%$ cases, "no resolution" in $4.8 \%$, "worsening of symptoms" was present in $2.1 \%$. These are the patients in which causes other than motility and non-motility disorders, association with $\mathrm{H}$ pylori should be sorted out i.e. psychic social factors.

From the literature review, we came to know that psycho social factors play an important role in the pathogenesis of NUD. Such patients have reduced visceral threshold for pain perception. Sense of having dyspeptic symptoms most of the time, frequents visits to health care providers and financial aspects all bring much psychological stress and anxiety. In fact, in the Netherlands, it is shown those women who are divorced, unemployed, or have incapacitated psychological distress, have higher chance of visiting the doctor. A recent study established that dyspeptic patients in general have higher level of psychological distress, whether or not they consult a doctor. Patients who consult the doctor as compared with non- consulters have more intense and longer lasting symptoms and are more likely to be a female. Health values and attention seeking behaviors clearly play a vital role in this process. The task of the doctor dealing with patients with high probability of psychological distress should therefore not be primarily directed towards relief of dyspeptic symptoms, to $\mathrm{H}$ pylori eradication but should also be towards relief of anxiety and depressive illnesses.

There is no consensus regarding eradication of $\mathrm{H}$ pylori influences symptoms in NUD or not. Data from international studies revealed conflicting results. Although meta-analysis of 10 studied failed to demonstrate an improvement in the symptoms with eradication therapy but an updated review of 17 trials revealed small but statistically significant benefit. ${ }^{13-14}$ The latest review related to eradication of $\mathrm{H}$. pylori for non-ulcer dyspepsia done in 2009 (the Cochrane Collaboration) revealed that $\mathrm{H}$. pylori eradication therapy improve symptoms in patients with NUD. The impact on symptoms was statistically significant but the effect size was small, not long lasting and most $\mathrm{H}$. pylori positive patients with NUD will still have symptoms after eradication therapy.

Our study results are in accordance with the outcomes of "Cochrane review" because in our study the response to eradication was $61 \%$ thereby supporting the use of eradication treatment in symptomatic patients with NUD.

Our results are comparable with Cadet Hp study which was conducted at Canada where response to eradication was $50 \%$ as compared with $36 \%$ in placebo group at 12 months. We studied the quality of life measures including daily symptoms, severity of symptoms in terms of mild, moderate and severe and hours off work. We found that NUD is responsible for affecting the quality of life; daily symptoms were present in $38.4 \%$ of patients, mild symptoms in $50.0 \%$ and are also responsible for some loss of work, with a mean 
1.455. Similar results were seen in Saudi study in which impact of NUD on health related quality of life was assessed in terms of frequency and severity of symptoms, though we did not assess the psychological aspects in NUD.

Non-responders to eradication were $38.4 \%$ out of which $31.4 \%$ showed symptomatic improvement in response to PPI. These results are in contrast to one seen in Chinese study to assess the efficacy of different doses of PPI or placebo for the management of functional dyspepsia in 453 patients for four weeks. Dyspepsia symptom scores and quality of life (SF-36 score) were evaluated before and four weeks after treatment. It was found that PPI therapy was not better than placebo for the management of functional dyspepsia in Chinese patients. ${ }^{12}$

\section{Our study has got few limitations}

The cross sectional design of the study limits elucidation of the results. Firstly, our study included population representative mainly of military origin and most of them were not the permanent residents of Quetta. They had superior fitness level and better socioeconomic background in general as compared to common civilian inhabitant of same age and socioeconomic background. Thus, selective response seems likely in our study group.

Secondly, findings can only be generalized towards dyspeptic patients visiting a doctor. We were not able to include a control group of non-dyspeptic consulters or dyspeptic non - consulters which might have added useful information to the result of the study.

Thirdly, we blindly gave $\mathrm{H}$. pylori eradication therapy without doing antral biopsy and confirming it on histopathological examination. Fourthly, we only studied the immediate effect of treatment on dyspeptic symptoms in patients with recurrent chronic dyspepsia. Recurrence of symptoms that may appear later on needs to be studied further.

Our findings have a few clinical implications for the treatment of patients with NUD. In accordance with results of previous studies that have shown that eradicating $\mathrm{H}$. pylori is beneficial in patients with NUD, we also found that eradication of this infection is beneficial.

Upper Gl endoscopy should be reserved for patients with chronic recurring symptoms.

\section{CONCLUSION}

Dyspepsia is a major health problem worldwide and specially in the developing countries like Pakistan where it put a lot of financial burden on national economy. The prescriptions for dyspepsia now account for over $100 \%$ in primary care. This is the commonest type of dyspepsia encountered in primary care and gastroenterology practice .Our study suggests that $\mathrm{H}$ pylori eradication therapy improves symptoms in patients with NUD. The impact on symptoms was significant. Thus $\mathrm{H}$ pylori eradication therapy is cost effective. Proton pump inhibitors are valuable in short-term treatment for non-ulcer dyspepsia in those who don't respond to eradication therapy. We also suggest

Reassurance and counseling

Copyright $@ 21$ June, 2016.

\section{REFERENCES}

1. Torpy JM, Lynm C, Glass RM. Dyspepsia. JAMA 2006 Apr; 295(13):1612.

2. Khan N, Shabbir G, Zarif M, Khattak Ml. Upper gastrointestinal Endoscopic assessment of patients presenting with dyspepsia. J Postgrad Med Inst 2OO7Sep; 21(3):212-6.

3. Yaqoob N, Malik JM. Nonulcer dyspepsia: a diagnostic optimism but therapeutic pessimism. Fauji Found Health J 2001 Mar; 2(1):17-19.

4. Talley NJ, Boyce P, Jones M. Dyspepsia and health care seeking in a community: How important are Psychological factors? Dig Dis Sci 1998 May; 43(5):1016- 22.

5. Mahmood K, Saeedi Ml, Mohammad R, Zia ud Din. Upper gastrointestinal Endoscopic findings in patients with dyspepsia. J Postgrad Med Inst 2006; 20(1):70.

6. Selgrad M, Kandulski A, Malfertheiner P. Dyspepsia and Helicobacter pylori. Dig Dis 2008 May; 26(3):210-4.

7. Gilvarry $\mathrm{J}$, Buckley $\mathrm{MJ}$, Beattie $\mathrm{S}$, Hamilton $\mathrm{H}$, O'Morain CA. Eradication of Helicobacter pylori affects symptoms in non-ulcer dyspepsia. Scand $J$ 
Gastroenterol 1997 Jun; 32(6):535-40.

8. Friedman LS. Helicobacter pylori and non-ulcer dyspepsia. N Engl J Med 1998; 339:1928-30.

9. Blum AL, Talley NJ, O'Morain C. Lack of effect of treating Helicobacter pylori infection in patients with non-ulcer dyspepsia. N Engl J Med 1998; 339:187581.

10. AlMalki AS. Helicobacter pylori eradication in nonulcer dyspepsia: Does it really matter? Saudi J Gastroenterol 2008; 14:93- 5.
11. Ahuja V. The case for Helicobacter pylori eradication in India: sensationalism, skepticism and scientific salesmanship. Indian J Gastroenterol 2006; 25:20-4.

12. Wong WM, Wong BC, Hung WK. Double Blind, Randomized, placebo controlled study of four weeks of lansoprazole for the treatment of functional dyspepsia in Chinese patients. Gut 2002; 51:502-6.

13. Talley NJ. Absence of benefit of eradicating Helicobacter pylori in patients with non-ulcer dyspepsia. $N$ Engl $J$ Med 2000; 342:589-90.

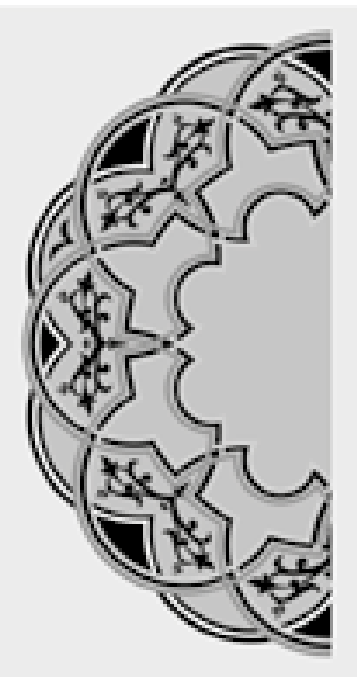

\section{"No storm lasts forever. The sunshine always returns."}

Unknown

AUTHORSHIP AND CONTRIBUTION DECLARATION

\begin{tabular}{|c|c|c|c|}
\hline Sr. \# & Author-s Full Name & Contribution to the paper & Author $=s$ Signature \\
\hline 1 & Dr. Farrukh Zahra & Done research main writer & \\
\hline & Dr Muhammad Younis & $\begin{array}{l}\text { Helped in writing the } \\
\text { article }\end{array}$ & 2 \\
\hline 3 & Dr. Uzma Nisar & $\begin{array}{l}\text { Helped in writing, } \\
\text { Collection or Ref. }\end{array}$ & $|y-2|$ \\
\hline
\end{tabular}

